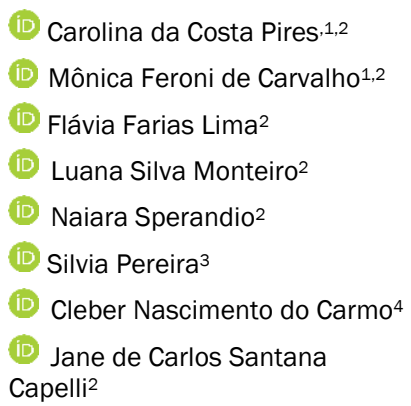

1 Prefeitura Municipal de Macaé, Secretaria Municipal de SaúdeMacaé, RJ, Brasil.

2 Universidade Federal do Rio de Janeiro, Curso de Nutrição. Campus UFRJ-Macaé Professor Aloisio Teixeira. Macaé, RJ, Brasil.

3 Universidade Federal Fluminense, Faculdade de Nutrição. Niterói, RJ, Brasil.

${ }^{4}$ Fundação Oswaldo Cruz, Escola Nacional de Saúde Pública Sérgio Arouca, Departamento de

Epidemiologia e Métodos Quantitativos em Saúde. Rio de Janeiro, RJ, Brasil.

Correspondência

Carolina da Costa Pires

c_pires4@hotmail.com

\section{Evolução do excesso de peso em gestantes usuárias da Atenção Primária à Saúde do município de Macaé-RJ entre 2010-2018}

\author{
Evolution of overweight pregnant women in the Primary \\ Health Care in the city of Macaé-RJ between 2010-2018
}

\section{Resumo}

Introdução: O estudo visa investigar a taxa de prevalência e a tendência temporal do excesso de peso em gestantes usuárias da Atenção Primária à Saúde de Macaé, entre 2010 e 2018. Método: Realizou-se estudo quantitativo, de série temporal, com a base de dados do Sistema de Vigilância Alimentar e Nutricional (Sisvan WEB), de acesso público, referente ao diagnóstico nutricional de gestantes $\geq 20$ anos usuárias da Atenção Primária à Saúde do município de Macaé, no período de 2010 a 2018. Analisaram-se 4.279 registros de gestantes no período de nove anos. As variáveis estudadas foram: ano de coleta e registro dos dados; baixo peso; peso adequado; sobrepeso; obesidade; excesso de peso (sobrepeso e obesidade). A análise de tendência do excesso de peso foi realizada por meio de modelos de regressão linear simples, considerando-se o nível de significância de 5\%. Resultados: As prevalências do excesso de peso em gestantes foram elevadas e ascendentes: 2010 (40,2\%), 2011 (37,5\%), 2012 (46,8\%), 2013 (48,8\%), 2014 (50,9\%), 2015 (51,8\%), 2016 (54,4\%), 2017 (57,6\%) e 2018 (55,8\%). Em todas as análises, detectou-se a tendência significativa e crescente, podendo-se destacar o desfecho do excesso de peso, que apresentou velocidade de incremento anual ( $\beta 1=2,35$; $p$-valor $<0,001)$ superior aos demais desfechos analisados em separado. Conclusão: Conclui-se que é elevada e crescente a prevalência do excesso de peso em gestantes usuárias da Atenção Primária à Saúde do município de Macaé no período estudado.

Palavras-chave: Excesso de Peso. Gestantes. Série Temporal. 
(40.2\%), 2011 (37.5\%), 2012 (46.8\%), 2013 (48.8\%), 2014 (50.9\%), 2015 (51.8\%), 2016 (54.4\%), 2017 (57.6\%) and 2018 (55.8\%). All analyses showed a significant and growing trend, especially for the excess weight outcome, whose annual increase speed $(£ 1=$ 2.35; p-value <0.001) was higher than the other outcomes, when analyzed separately. Conclusion: Prevalence of excess weight in pregnant women receiving Primary Health Care in the city of Macaé was high and growing during the study period.

Keywords: Excess weight. Pregnant women. Time Series. 


\section{INTRODUÇÃO}

O excesso de peso, compreendido como sobrepeso e obesidade, tem apresentado elevada e crescente prevalência nas últimas décadas, constituindo relevante problema de saúde pública que acomete países desenvolvidos e em desenvolvimento. ${ }^{1}$

Dados de 2012 da International Association for the Study of Obesity estimavam quase 1,5 bilhão de adultos com excesso de peso no mundo, dos quais 475 milhões apresentavam seu estágio mais avançado, a obesidade. ${ }^{2}$ Em 2016, a Organização Mundial da Saúde (OMS) atualizou tais dados para 1,9 bilhão de pessoas com excesso de peso, correspondendo a 39,0\% dos adultos do mundo com excesso de peso, das quais 650 milhões com obesidade. ${ }^{3}$

No Brasil, de acordo com as pesquisas representativas de âmbito nacional realizadas pelo Instituto Brasileiro de Geografia e Estatística (IBGE) entre 1974 e 2009, o excesso de peso em adultos ( $\geq 20$ anos) quase triplicou em homens (de 18,5 para 50,1\%) e quase dobrou em mulheres (de 28,7 para 48,0\%), atingindo prevalências muito próximas. ${ }^{4,5} \mathrm{Na}$ década subsequente, diante da ausência da continuidade dessas pesquisas pelo IBGE, as prevalências do Estudo de Vigilância de Fatores de Risco e Proteção para Doenças Crônicas por Inquérito Telefônico (Vigitel), realizado apenas em regiões metropolitanas brasileiras, apontaram a evolução crescente de 51,0\%, 52,5\%, 53,8\% e 54,0\%, para os anos de 2012, 2014, 2016 e 2018, respectivamente. ${ }^{6}$

Na população feminina, essa tendência de aumento do excesso de peso se manteve como sinalizado nas seguintes pesquisas: Pesquisa Nacional de Demografia e Saúde (PNDS) de 2006 revelou 43,0\% de excesso de peso na população feminina; a última Pesquisa de Orçamentos Familiares (POF 2008-2009), 48,0\%,e o último Vigitel (2018), 53,9\%. No Vigitel 2018, a Região Metropolitana do Rio de Janeiro obteve a maior prevalência feminina de excesso de peso, atingindo $58,4 \% .^{7-9}$

A elevada prevalência do excesso de peso também é verificada no período gestacional, estando em torno de 25,0 a 30,0\%, ${ }^{10-12}$ considerando-se um dos fatores de risco mais presentes na prática obstétrica. ${ }^{13-}$ 15 No âmbito da saúde materna, essa prevalência é preocupante, uma vez que a mulher em período gestacional apresenta necessidades nutricionais aumentadas que, não sendo monitoradas adequadamente, poderão intensificar o ganho de peso gravídico. ${ }^{16-18}$ Além disso, a obesidade no período gestacional está associada às morbidades como diabetes gestacional, distúrbios hipertensivos, intercorrências cardiovasculares, eventos tromboembólicos, cesarianas e complicações cirúrgicas no parto; e os neonatos têm maiores chances de apresentarem malformações congênitas, escore de Apgar baixo, macrossomia, hipoglicemia e eventual morte neonatal.5,19

Apesar da existência de protocolos clínicos de cuidado nutricional e recomendação de ganho ponderal de acordo com a condição nutricional prévia, é cada vez mais comum o excesso de peso gestacional, fenômeno facilitado por construções socioculturais que encorajam a adoção de práticas alimentares inadequadas - o "comer por dois" e o enaltecimento da barriga como valorização da vida e feminilidade. ${ }^{16,20-}$ ${ }^{23}$ Torna-se, portanto, fundamental a vigilância nutricional na gestação, uma vez que o acúmulo anormal ou excessivo de peso pode comprometer a saúde materno-fetal. ${ }^{16-18}$

No município de Macaé, situado na região norte do Estado do Rio de Janeiro, há a vigilância nutricional de gestantes através do Sistema de Vigilância Alimentar e Nutricional (Sisvan), contudo, somente a partir de 2008 foram ampliados os esforços da gestão do município no monitoramento do estado nutricional nessa fase do curso da vida. 
Diante da inexistência de estudos epidemiológicos nessa área e da relevância do tema, o presente estudo tem como objetivo investigar a taxa de prevalência e a tendência temporal do excesso de peso em gestantes atendidas na Atenção Primária à Saúde (APS) do município de Macaé, entre 2010 e 2018.

\section{MÉTODOS}

Estudo quantitativo, de série temporal, que utilizou a base de dados do Sisvan Web, referentes ao diagnóstico nutricional de gestantes $\geq 20$ anos usuárias da APS, do município de Macaé, no período entre 2010 e 2018. Este município da região norte fluminense do Estado do Rio de Janeiro tem sua economia baseada na exploração do petróleo e outros combustíveis fósseis, comércio, formação acadêmica e turismo. ${ }^{24}$ Possui população estimada de 256.672 pessoas, 48,9\% ocupados com trabalho e rendimento médio mensal de 6,4 salários mínimos entre os trabalhadores formais. Apesar disso, apresenta 31,5\% da população com rendimento mensal de <1/2 salário mínimo/pessoa. ${ }^{25}$

Na série temporal do estudo, foram registrados os dados de 4.279 gestantes pelo Sisvan Web. No primeiro ano da série temporal (2010), 262 gestantes e, ao final (2018), 677. Destaca-se a oscilação do número de registros anuais, sobretudo no ano de 2015, quando se notou redução dos acompanhamentos para 197 gestantes; no entanto, essas oscilações não interferiram nos resultados, uma vez que foram analisadas as proporções.

Os dados foram acessados no mês de outubro de 2019, por meio dos relatórios gerados pelo sítio eletrônico do Sisvan Web, utilizando o acesso restrito 26 pelo link<http://sisaps.saude.gov.br/sisvan/relatoriopublico/index>.As variáveis do estudo foram: (a) ano de coleta e registro dos dados; (b) baixo peso; (c) adequado; (d) sobrepeso; (e) obesidade; (f) excesso de peso. As variáveis (b) a (e) foram geradas pelo Sisvan Web nos relatórios de diagnóstico nutricional pelo índice de massa corporal/ idade gestacional, com base na curva de Atalah, que é recomendada pelo Ministério da Saúde. ${ }^{27}$ A variável (f) excesso de peso foi a somatória das variáveis (d) e (e).

O banco de dados foi construído no software Microsoft Excel for Windows ${ }^{\circledR}$ versão 2010 e analisado no software Statistical Package for the Social Sciences (SPSS) versão 20.0®. Na análise descritiva, os dados foram caracterizados por suas frequências absolutas e percentuais. As diferenças entre frequências anuais e entre as categorias de diagnóstico do índice de massa corporal/ idade gestacional (em proporções) foram verificadas pelo teste de qui-quadrado. A análise de tendência do excesso de peso foi realizada por meio de modelos de regressão linear simples, segundo a equação: $Y=\beta_{0}+\beta_{1} X$, em que $\beta_{0}$ poderia ser interpretada como uma prevalência anual média e $\beta_{1}$ representaria um efeito linear (velocidade do acréscimo ou decréscimo ao longo do tempo). Foram utilizadas proporções em relação ao total de dados coletados em cada ano para gerar as prevalências. Considerou-se tendência significativa aquela em que o modelo estimado apresentou p-valor significativo. O nível de significância de 5\% foi considerado em todas as análises.

Foram consideradas como variável desfecho $(Y)$, separadamente, as prevalências anuais de sobrepeso, obesidade e de excesso de peso e como variável independente $(X)$, os anos de estudo. A transformação da variável ano de coleta dos dados na variável ano-centralizada (ano menos o ponto mediano do período de estudo) fez-se necessária, já que em modelos de regressão linear com dados monitorados ao longo do tempo, os termos da equação frequentemente são altamente correlacionados, e expressar a variável independente como um desvio de sua mediana reduz substancialmente a autocorrelação entre eles.

Por se tratar de um estudo com dados secundários, de domínio público e com a autorização do nível central, sem a possibilidade de identificação dos sujeitos, em acordo com a Resolução n 466/2012 do 
Conselho Nacional de Saúde/Ministério da Saúde, ${ }^{28}$ não necessitou de apreciação por um Comitê de Ética em Pesquisa.

\section{RESULTADOS}

A tabela 1 apresenta a distribuição percentual de 4.279 gestantes ( $\geq 20$ anos) usuárias da APS de Macaé, segundo ano e índice de massa corporal por semana gestacional (IMC/idade gestacional), entre 2010 e 2018, observando-se significância estatística entre as prevalências anuais, segundo categorias de $\operatorname{IMC}(p<0,001)$.

Cabe destacar que as prevalências do excesso de peso até 2015 estavam abaixo de 10,0\%, e a partir de 2016, ficaram acima desse percentual: 2016 (16,9\%), 2017 (20,8\%), 2018 (17,4\%), conforme a tabela 1.

Tabela 1. Distribuição percentual do índice de massa corporal gestacional (IMC/semana Gestacional) segundo ano, de gestantes $\geq 20$ anos, usuárias da Atenção Primaria à Saúde de Macaé, 2010-2018. Sisvan Web ( $n=4.279)$.

\begin{tabular}{ccccccc}
\hline Ano & \multicolumn{5}{c}{ IMC gestacional } \\
& $\begin{array}{c}\text { Baixo-Peso } \\
\mathrm{n}(\%)\end{array}$ & $\begin{array}{c}\text { Adequado } \\
\mathrm{n}(\%)\end{array}$ & $\begin{array}{c}\text { Sobrepeso } \\
\mathrm{n}(\%)\end{array}$ & $\begin{array}{c}\text { Obesidade } \\
\mathrm{n}(\%)\end{array}$ & $\begin{array}{c}\text { Excesso de Peso* } \\
\mathrm{n}(\%)\end{array}$ & $\begin{array}{c}\text { Total } \\
\mathrm{n}(\%)\end{array}$ \\
\hline 2010 & $67(10,7)$ & $91(6,1)$ & $61(5,2)$ & $45(4,5)$ & $106(4,9)$ & $264(6,2)$ \\
2011 & $114(18,2)$ & $218(14,7)$ & $109(9,3)$ & $90(9,0)$ & $199(9,2)$ & $531(12,4)$ \\
2012 & $71(11,4)$ & $121(8,1)$ & $95(8,1)$ & $74(7,4)$ & $169(7,8)$ & $361(8,4)$ \\
2013 & $63(10,1)$ & $150(10,1)$ & $112(9,6)$ & $91(9,1)$ & $203(9,4)$ & $416(9,7)$ \\
2014 & $51(8,2)$ & $134(9,0)$ & $110(9,4)$ & $82(8,2)$ & $192(8,9)$ & $377(8,8)$ \\
2015 & $24(3,8)$ & $71(4,8)$ & $50(4,3)$ & $52(5,2)$ & $102(4,7)$ & $197(4,6)$ \\
2016 & $79(12,6)$ & $228(15,3)$ & $204(17,5)$ & $162(16,2)$ & $366(16,9)$ & $673(15,7)$ \\
2017 & $83(13,3)$ & $249(16,7)$ & $229(19,6)$ & $222(22,2)$ & $451(20,8)$ & $783(18,3)$ \\
2018 & $73(11,7)$ & $226(15,2)$ & $198(17,0)$ & $180(18,0)$ & $378(17,4)$ & $677(15,8)$ \\
\hline Total & $625(100,0)$ & $1.488(100,0)$ & $1.168(100,0)$ & $998(100,0)$ & $2.166(100,0)$ & $4.279(100,0)$ \\
\hline
\end{tabular}

*Excesso de peso = sobrepeso + obesidade.

p-valor <0,001 (diferença estatística observada em todas as categorias analisadas).

Ao analisar a prevalência do excesso de peso em cada ano estudado, detectaram-se 40,2\% em 2010; em 2011, 37,5\%; em 2012, 46,8\%; em 2013, 48,8\%; em 2014, 50,9\%; em 2015, 51,8\%; em 2016, 54,4\%; em 2017, 57,6\%; em 2018, 55,8\% (dados não apresentados em tabela).

A figura 1 apresenta a tendência do excesso de peso, sobrepeso e obesidade no período estudado. Em todas as análises, verificou-se tendência significativa e crescente do fenômeno estudado. Destaque deve 
ser dado ao desfecho excesso de peso (ep), que apresentou velocidade de incremento anual ( $\beta 1=2,35 ; \mathrm{p}$ valor $<0,001$ ) superior aos demais desfechos analisados separadamente (figura 1).

Figura 1. Tendência do excesso de peso, sobrepeso e obesidade em nove anos de acompanhamento de gestantes $\geq$ 20 anos, usuárias da Atenção Primária à Saúde de Macaé, 2010-2018. Sisvan Web (n=4.279).

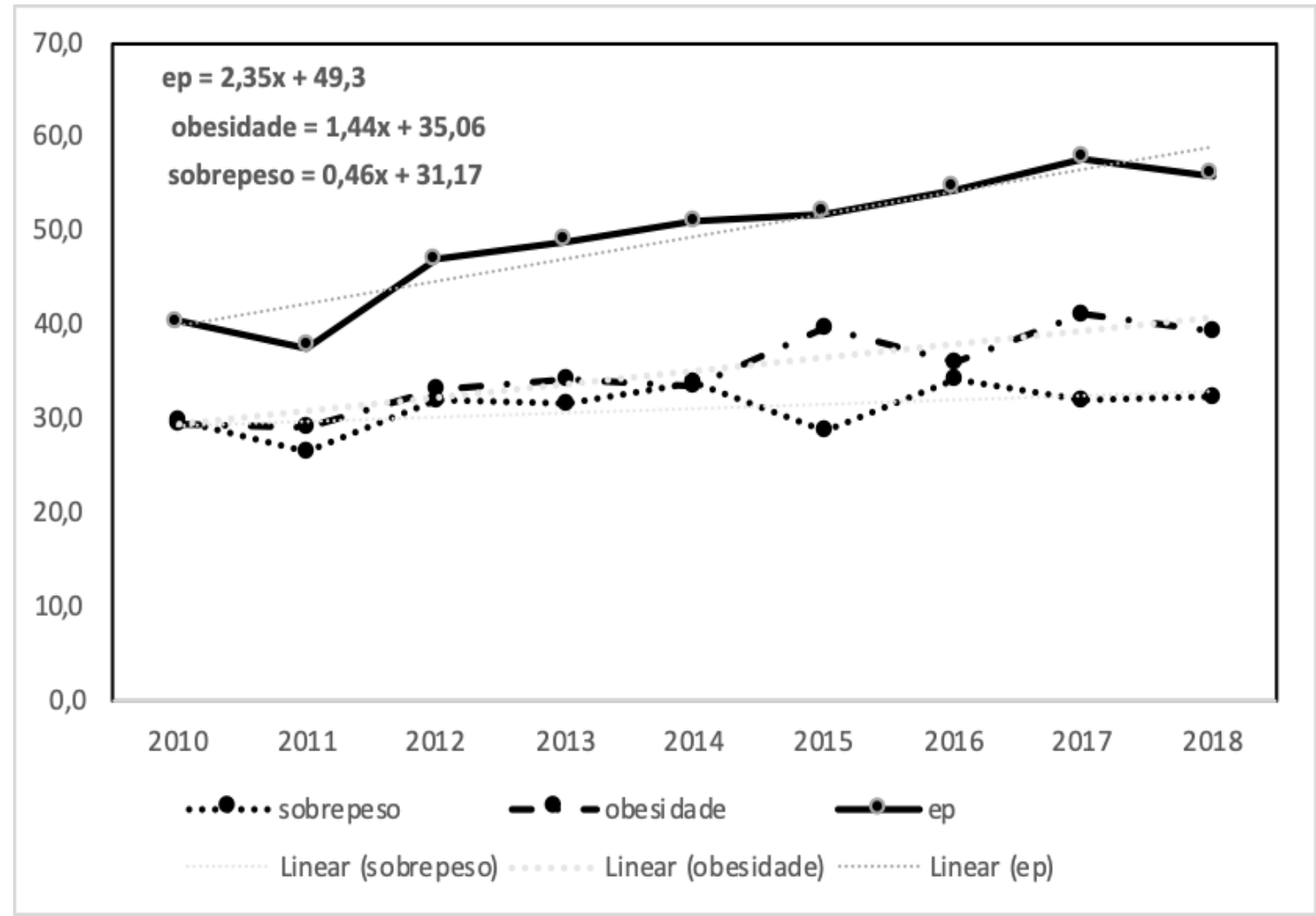

Nota: $\mathrm{p}$-valor $<0,001$.

\section{DISCUSSÃO}

Neste estudo, as prevalências do excesso de peso em gestantes foram elevadas, e a tendência excesso de peso no município de Macaé foi significativa e crescente no período estudado.

Os resultados encontrados alinham-se ao cenário epidemiológico mundial, o qual evidencia o número crescente de indivíduos apresentando excesso de peso. Como destacado no relatório da Comissão The Lancet, $^{29}$ o excesso de peso corporal afeta mais de 2 bilhões de pessoas no mundo todo, e os custos econômicos atuais da obesidade são estimados por volta de $2,8 \%$ do produto interno bruto (PIB) mundial. ${ }^{29}$ Diante dessa realidade, o relatório revela que enfrentamos um dos principais desafios de nossa era, e traz o novo conceito de "sindemia global", destacando as três pandemias (obesidade, desnutrição e mudanças climáticas) inter-relacionadas, que compartilham causalidades e exercem interferência mútua sobre a sociedade. ${ }^{29}$

Esse cenário é preocupante entre as gestantes, uma vez que a literatura aponta a associação do excesso de peso gestacional ao maior desenvolvimento de diabetes, pré-eclâmpsia e retenção de peso materno. Além disso, está relacionado à macrossomia fetal e excesso de peso ao nascer, que podem alterar substancialmente o estado nutricional e a saúde infantil. ${ }^{30}$ 
Não foram encontrados estudos de tendência no Brasil apontando a contribuição e originalidade dos resultados. No entanto, destacam-se estudos seccionais, como o de Furlan e colaboradores, ${ }^{31}$ investigando o diagnóstico nutricional de 52 gestantes atendidas em unidades básicas de saúde da região norte do Rio Grande do Sul, no ano de 2018, que revelou 30,8\% de sobrepeso e 28,8\% de obesidade, somando 59,6\% de prevalência de excesso de peso em gestantes. No mesmo ano, o presente estudo obteve prevalência de $55,8 \%$.

O estudo desenvolvido por Silva e colaboradores, ${ }^{32}$ com 298 puérperas internadas em uma maternidade de Joinville-SC entre maio e junho de 2013, encontrou 28,1\% ( $n=84$ ) de sobrepeso e 17,8\% $(n=53)$ de obesidade, somando 45,9\% de prevalência de excesso de peso. No mesmo ano, Shub e colaboradores ${ }^{33}$ obtiveram prevalência de $50,0 \%$ das gestantes avaliadas com excesso de peso. O presente estudo obteve prevalência de 48,8\% em 2013, algo semelhante aos estudos mencionados.

Nunes, ${ }^{13}$ em estudo transversal com 3.405 puérperas da Região Sudeste, utilizando dados de uma coorte nacional entre 2011 e 2012, com base hospitalar denominada "Nascer no Brasil: inquérito nacional sobre parto e nascimento", encontrou cerca de 31,0\% de mulheres com excesso de peso pré-gestacional (sobrepeso e obesidade).

Nucci e colaboradores ${ }^{10}$ encontraram prevalência de 24,7\% em gestantes usuárias do Sistema Único de Saúde (SUS) de seis capitais brasileiras entre 1991 e 1995, enquanto Fonseca e colaboradores ${ }^{34}$ encontraram 34,7\% de excesso de peso pré-gravídico e 36,9\% de ganho de peso excessivo em gestantes internadas para o parto em Jundiaí-SP.

O único estudo encontrado em Macaé, realizado por Diniz e colaboradores ${ }^{35}$ em um hospital público de referência do município, no segundo semestre de 2014, detectou 41,7\% das puérperas com excesso de peso no início da gestação (15,6\% sobrepeso e 26,1\% obesidade) e 34,8\% de ganho de peso excessivo na gestação.

Os achados apontam um cenário preocupante do excesso de peso, tanto em gestantes de diferentes regiões do país como nas de Macaé. Uma hipótese levantada foi sobre a autopercepção da imagem corporal da mulher na condição gravídica. Estudo conduzido por Pires e colaboradores ${ }^{21}$ com gestantes apresentando o diagnóstico nutricional de excesso de peso, assistidas em duas unidades da APS do município de Macaé no ano de 2017, verificou a "naturalização" e "resignação" das entrevistadas ao peso gestacional excessivo, em função da condição gravídica e em prol da saúde do bebê.

Nesse campo, encontra-se o estudo de Shub e colaboradores, ${ }^{33}$ que revelou $24,0 \%$ das gestantes se identificando com excesso de peso, ou seja, a maioria delas considerava seu peso normal, 30 resultado que corrobora as construções socioculturais de encorajamento à naturalização do excesso de peso e das práticas alimentares inadequadas durante a gestação, além da não adesão e continuidade da atenção nutricional pré-natal. ${ }^{21,22}$

Nessa direção, o monitoramento do estado nutricional se torna imprescindível nas mulheres em idade fértil, uma vez que o excesso de peso também é verificado no período pré-gravídico e agravado pelas práticas alimentares inseguras em qualidade e quantidade no período gestacional.4,9,22 O acréscimo de várias refeições ao longo do dia, justificado pelo incremento das necessidades nutricionais ("comer por dois") e fatores emocionais ("desejos"), permeia o cotidiano de gestantes e é incentivado pelo companheiro e familiares. ${ }^{21}$

A utilização dos dados provenientes do Sisvan Web constitui importante ferramenta da Vigilância Alimentar e Nutricional, sendo fundamental para a formulação e avaliação de políticas públicas em 
alimentação e nutrição, e organização da atenção nutricional em todos os cursos de vida, inclusive gestantes..$^{27,37}$ Contudo, algumas limitações devem ser consideradas na análise de dados consolidados de base secundária, tais como: a qualidade dos dados registrados (falta de padronização na coleta e digitação);38 a ausência de informação para alguns parâmetros relevantes à análise de interesse (idade materna, peso pré-gestacional, paridade e semana gestacional no momento das medidas corporais, por exemplo) ${ }^{27}$ e a variação da cobertura dos dados ao longo do tempo..$^{39-42}$

Nessa perspectiva, pode ser mencionada como limitação do estudo a oscilação na cobertura dos acompanhamentos ao longo dos anos. A fragilidade dos vínculos empregatícios e a alta rotatividade das equipes de saúde da APS, especialmente no ano de 2015, diminuíram a cobertura da Vigilância Alimentar e Nutricional no município de Macaé, ${ }^{42}$ achado semelhante àquele verificado por Rolim e colaboradores. ${ }^{43}$ Entretanto, apesar das limitações mencionadas, o baixo custo para a obtenção dos dados e a possibilidade de análises temporais da informação ${ }^{36,40}$ constituem vantagens quando os relatórios consolidados do Sisvan são utilizados. ${ }^{40,41,43}$

Devido à crescente importância atribuída à informação produzida pelos Sistemas de Informação em Saúde, faz-se necessário, portanto, que esses sistemas não sejam negligenciados pelos gestores e esforços sejam mobilizados para a qualificação dessas informações e melhoria dos indicadores em saúde, sobretudo no âmbito da Alimentação e Nutrição. ${ }^{27,40-44}$

Além disso, cabe ressaltar que a obesidade tem sido importante objeto de intervenção no campo das políticas públicas no Brasil, podendo-se destacar a Política Nacional de Alimentação e Nutrição (publicada em 1999 e revisada em 2012), que, por meio de suas diretrizes no âmbito do SUS, e da Rede de Atenção à Saúde das Pessoas com Doenças Crônicas, tem buscado reorganizar e orientar ações voltadas a sua prevenção e tratamento na população. ${ }^{45}$

No campo da Saúde Materna, em 2018 foi publicada a 4ª edição da Caderneta da Gestante pelo Ministério da Saúde, ${ }^{46}$ importante instrumento de acompanhamento da assistência pré-natal que, dentre seus objetivos, apresenta o acompanhamento nutricional, principalmente o monitoramento do ganho de peso gestacional. Está coadunada à Rede Cegonha, ${ }^{47} \mathrm{Cuja}$ finalidade é implementar uma rede de cuidados que assegurem a todas as mulheres brasileiras o direito ao planejamento reprodutivo e a atenção humanizada na gravidez, parto e puerpério, aborto e às crianças menores de dois anos, bem como estruturar e organizar a atenção à saúde materno-infantil no Brasil.

No entanto, toda essa estrutura precisa ter desde a sensibilização e qualificação profissional, até a produção de dados fidedignos ${ }^{38}$ que alimentem a base de dados do Sisvan. A partir da divulgação das informações, 37-40 os gestores devem buscar soluções e subsidiar ações para o binômio obesidade/gestação, o que é um fato em nossa sociedade e repercute negativamente na saúde da mulher e do concepto. Devem, ainda, ampliar os investimentos na reorganização dos serviços de saúde, no que tange a rede e linhas de cuidados da obesidade, ${ }^{45}$ assim como na assistência pré-natal, por meio de ações do Ministério da Saúde, como a Rede Cegonha.

\section{CONCLUSÃO}

Neste estudo, os achados mostram a elevada e crescente prevalência do excesso de peso em gestantes atendidas na Atenção Primária de Saúde do município de Macaé. É importante que ações e estratégias no campo das políticas públicas voltadas ao grupo materno, que está exposto a diferentes fatores 
relacionados ao ganho e excesso de peso, sejam pensadas, de modo a reverter o cenário da população de gestantes daquele município.

\section{REFERÊNCIAS}

1. World Health Organization.Obesity and overweight; 2019.[Acesso em 110ut 2019]. Disponível em: https://www.who.int/features/factfiles/obesity/en/.

2. International Association for the Study of Obesity. Obesity the Global Epidemic. Obesity \& Research; 2012.[Acesso em 11out 2019].Disponível em: https://www.iaso.org/iotf/obesity/obesitytheglobalepidemic/.

3. Organização Pan-Americana da Saúde. Organização Mundial da Saúde. OPAS Brasil. OPAS/OMS Reúnem especialistas para apoiar Brasil na avaliação de impacto econômico de medidas que beneficiam saúde da população; 2019[Acesso em 11 out 2019].Disponível em: https://www.paho.org/bra/index.php?option=com_content\&view=article\&id=5699:opas-oms-reuneespecialistas-para-apoiar-brasil-na-avaliacao-de-impacto-economico-de-medidas-que-beneficiam-saude-dapopulacao\&ltemid=839.

4. Instituto Brasileiro de Geografia e Estatística. Pesquisa de Orçamentos Familiares 2008-2009. Antropometria e estado nutricional de crianças, adolescentes e adultos no Brasil. Rio de Janeiro: IBGE; 2010.

5. Correia LL, Silveira DMI, Silva CA, Machado TMM, Rocha LHA, et al. Prevalencia e determinantes de obesidade e sobrepeso em mulheres em idade reprodutiva residentes na região semiárida do Brasil. Ciênc Saúde Coletiva. 2011;16(1):133-145. https://doi.org/10.1590/S1413-81232011000100017

6. Ministério da Saúde. Secretaria de Vigilância em Saúde. Departamento de Vigilância de Doenças e Agravos não Transmissíveis e Promoção da Saúde. Vigitel Brasil 2016: vigilância de fatores de risco e proteção para doenças crônicas por inquérito telefônico: estimativas sobre frequência e distribuição sociodemográfica de fatores de risco e proteção para doenças crônicas nas capitais dos 26 estados brasileiros e no Distrito Federal em 2016. Brasília, DF: Ministério da Saúde; 2017.

7. Ministério da Saúde. Pesquisa Nacional de Demografia e Saúde da Criança e da Mulher 2006 (PNDS). Brasília: Ministério da Saúde; 2008.

8. Ministério da Saúde. Ministério do Planejamento, Orçamento e Gestão. Pesquisa de Orçamentos Familiares 2008-2009: antropometria e estado nutricional de crianças, adolescentes e adultos no Brasil. Instituto Brasileiro de Geografia e Estatística. Rio de Janeiro: IBGE; 2010.

9. Ministério da Saúde. Secretaria de Vigilância em Saúde. Departamento de Análise em Saúde e Vigilância de Doenças não Transmissíveis.Vigitel Brasil 2018: vigilância de fatores de risco e proteção para doenças crônicas por inquérito telefônico: estimativas sobre frequência e distribuição sociodemográfica de fatores de risco e proteção para doenças crônicas nas capitais dos 26 estados brasileiros e no Distrito Federal em 2018. Brasília: Ministério da Saúde; 2019.

10. Nucci LB, Schmidt MI, Duncan BB, Fuchs SC, Fleck ET, Britto MMS.Nutritional status of pregnant women: prevalence and associated pregnancy outcomes. RevSaúdePública.2001;35(6):502-507.https://doi.org/10.1590/S0034-89102001000600002

11. Stulbach TE, Benício MHD, Andreazza R, Kono S. Determinantes do ganho ponderal excessivo durante a gestação em serviço público de pré-natal de baixo risco. RevBrasEpidemiol. 2007;10(1):99-108. https://doi.org/10.1590/S1415$790 \times 2007000100011$

12. Vitolo MR, Bueno MSF, Gama CM. Impact of a dietary counseling program on the gain weight speed of pregnant women attended in a primary care service. Rev Bras Ginecol Obstet. 2011;33(1):13-9. https://doi.org/10.1590/S010072032011000100002

13. Nunes CTG. Análise do ganho de peso gestacional em mulheres da região sudeste do Brasil e desfechos perinatais. [dissertação]. Universidade de São Paulo. Faculdade de Saúde Pública.Programa de Pós-Graduação em SaúdePública. São Paulo; 2015.

14. Ruchat SM, Mottola MF. Preventing Long-Term Risk of Obesity for Two Generations: Prenatal Physical Activity Is Part of the Puzzle. Journal of Pregnancy 2012; 2012:1-33.https://doi.org/10.1155/2012/470247

15. Lim CC, Mahmood T. Obesity in pregnancy. Best Pract Res Clin Obstet Gynaecol 2015;29(3):309-319. https://doi.org/10.1016/j.bpobgyn.2014.10.008 
16. Ministério da Saúde. Secretaria de Atenção à Saúde. Departamento de Atenção Básica. Atenção ao pré-natal de baixo risco/Ministério da Saúde. Secretaria de Atenção à Saúde. Departamento de Atenção Básica.1ª. ed. Brasília, DF: Editora do Ministério da Saúde; 2013a.

17. Melere C,Hoffmann JL, Nunes MAA, Drehmer M, Buss C, Ozcariz SGI et al.Índice de Alimentação Saudável para Gestantes: Adaptação para uso em Gestantes Brasileiras. Rev Saúde Pública. 2013;47(1):20-8. https://doi.org/10.1590/S003489102013000100004

18. Nast M,Oliveira A,Rauber F,Vitolo MR. Ganho de peso excessivo na gestação é fator de risco para o excesso de peso em mulheres. RevBrasGinecolObstet. 2013;35(12):536-40.https://doi.org/10.1590/S0100-72032013001200002

19. Drehmer M, Camey S, Schmidt MI, Olinto MTA,Giacomello A, Buss C, et al. Fatores Socioeconômicos, demográficos e nutricionais associados com ganho de peso gestacional em unidades básicas de saúde no Sul do Brasil.Cad Saúde Pública. 2010;26(5):1024-1034.https://doi.org/10.1590/S0102-311X2010000500024

20. Loth KA, Bauer KW, Wall M, Berge J,Neumark-Sztainer D.Body satisfaction during pregnancy. Bodylmage, 2011;8(3):297300.https://doi.org/10.1016/j.bodyim.2011.03.002

21. Pires CC, Rodrigues ML, Capelli JC, Santos MMS, Baião MR. O excesso de peso na perspectiva de gestantes assistidas na atenção primária à saúde de um município da região do Norte Fluminense. Demetra 2018;13(3):555568.https://doi.org/10.12957/demetra.2018.33112

22. Pires, C.C. Sentidos do excesso de peso por gestantes adultas assistidas na Atenção Primária à Saúde de Macaé-RJ. [dissertação]. Rio de Janeiro: UFRJ/Faculdade de Medicina, Instituto de Atenção á Saúde São Francisco de Assis - HESFA; 2017.

23. Clark A, Skouteris H, Wertheim EH, Paxton SJ, Milgrom J. My baby body: A qualitative insight into women's body-related experiences and mood during pregnancy and the postpartum. J Reprod Infant Psychol.2009;27(4):330345.https://doi.org/10.1080/02646830903190904

24. Macaé. Governo do Estado do Rio de Janeiro. Brasil.[Acesso em 17 Out 2019].Disponível em:https://www.macae.rj.gov.br/conteudo/leitura/titulo/infraestrutura-completa.

25. Instituto Brasileiro de Geografia e Estatística - IBGE. Macaé.[Acesso em 17 out 2019].Disponível em: https://cidades.ibge.gov.br/brasil/rj/macae/panorama.

26. Ministério da Saúde. Datasus. Sisvan Web. [Acesso em 17 out 2019].Disponível em:https://sisaps.saude.gov.br/sisvan/relatoriopublico/index.

27. Ministério da Saúde. Secretaria de Atenção à Saúde. Orientações para a coleta e análise de dados antropométricos em serviços de saúde. Norma Técnica do Sistema de Vigilância Alimentar e Nutricional - SISVAN. Brasília, DF: Ministério da Saúde; 2011.

28. Ministério da Saúde. Conselho Nacional de Saúde. Resolução CNS n466, de 12 de dezembro de 2012. Contém as diretrizes e normas regulamentadoras de pesquisas envolvendo seres humanos. Brasília, DF; 2012a.

29. Swinburn BA, Kraak IV, Allender S, Atkins JV, Baker IP, Bogard RJ, et al.The Global Syndemic of Obesity, Undernutrition, and Climate Change:The Lancet Commission report. The Lancet Commissions.2019;393:791-846.https://doi.org/10.1016/S01406736(18)32822-8

30. Kashan AS, Kenny LC. The effects of maternal body mass index on pregnancy outcome. Eur J Epidemiol. 2009;24:697-705. https://doi.org/10.1007/s10654-009-9375-2

31. Furlan C, Carli G, Kumpel DA. Excesso de peso e consumo alimentar de gestantes atendidas em unidades básicas de saúde. Revista Saúde (Sta. Maria). 2019;45(2):1-12.https://doi.org/10.5902/2236583436625

32. Silva JC, Amaral AR, Ferreira BS, Petry JF, Silva, MR, Krelling PC.Obesidade durante a gravidez: resultados adversos da gestação e do parto.Rev Bras Ginecol Obstet.2014;36(11):509-513. https://doi.org/10.1590/S0100-720320140005024

33. Shub A, Huning EY, Campbell KJ, Mccarthy EA. Pregnant women's knowledge of weight, weight gain, complications of obesity and weight management strategies in pregnancy. BMC Res Notes. 2013;6:278.https://doi.org/10.1186/1756-0500-6-278

34. Fonseca MRCC, Laurenti R, Marin CR, Traldi MC. Ganho de peso gestacional e peso ao nascer do concepto: estudo transversal na região de Jundiaí, São Paulo, Brasil. CiêncSaúdeColetiva.2014;19(5):1401-1407. https://doi.org/10.1590/141381232014195.17022013

35. Diniz JGA, Paredes HDMT, Bouskelá A, Rocha CMM, Lima FF, Braga FAMN, et al. Estado nutricional materno e indicadores nutricionais associados ao peso ao nascer em um hospital de referência. In: Viera VB, Piovesan N, organizadoras. Inovação 
em ciência e tecnologia de alimentos 3 [recurso eletrônico] - Ponta Grossa, PR: Atena Editora, 2019. - (Inovação em Ciência e Tecnologia de Alimentos; v. 3).

36. Ministério da Saúde. Secretaria de Atenção à Saúde. Departamento de Atenção Básica. Política Nacional de Alimentação e Nutrição. $1^{\text {a }}$ ed., $1^{\text {a }}$ reimpr. Brasília, DF: Ministério da Saúde; 2013 b.

37. Ministério da Saúde. Secretaria de Atenção à Saúde. Departamento de Atenção Básica. Política Nacional de Atenção Básica. Brasília, DF: Ministério da Saúde; 2012b.

38. Silva GAS, Rocha CMM, Almeida MFL, Lima FF, Carmo CN,Boccolini CS, et al. Procedimentos de medição da massa corporal infantil pelos agentes comunitários de saúde de Macaé, Rio de Janeiro, 2010-2011. Epidemiol Serv Saúde.2017;26(3):579588.http://dx.doi.org/10.5123/s1679-49742017000300015

39. Coeli CM. Sistemas de Informação em Saúde e uso de dados secundários na pesquisa e avaliação em saúde. Cad Saúde Colet. 2010;18(3):335-6.

40. Pires CC,Capelli JCS, Rocha CMM, Miguel RR, Lima FF. O Profissional de Saúde e a Produção da Informação na Atenção Básica. In: Almeida MFL,Capelli JCS,Sperandio N, Rocha CMM, Ribeiro BG, organizadoras. Alimentação e Nutrição da Infância à Adolescência. $1^{\text {a }}$ ed. São Paulo: RED publicações, 2018. p.151-156.

41. Rocha CMM, Pires CC,Latorre CG, Araújo IFL. Sistemas de Informação em Saúde: Sisvan web. In: Almeida, MFL,CapellijCS, Sperandio N, RochaCMM, Ribeiro BG, organizadoras.Alimentação e Nutrição da Infância à Adolescência. $1^{\text {a }}$ ed. São Paulo: RED publicações, 2018. p.187-189.

42. Macaé. Governo do Estado do Rio de Janeiro. Brasil.[Acesso em 26 nov 2019].Disponível em: https://www.macae.rj.gov.br/midia/conteudo/arquivos/1434482884.pdf.

43. Rolim MD, LimaSML, Barros DC, Andrade CLT.Avaliação do SISVAN na gestão de ações de alimentação e nutrição em Minas Gerais, Brasil.Ciênc Saúde Coletiva.2015;20(8):2359-2369.http://dx.doi.org/10.1590/1413-81232015208.00902015

44. Drumond EF, Machado CJ, Vasconcelos MR, França F. Utilização de dados secundários do SIM, Sinasc e SIH na produção científica brasileira de 1990 a 2006. RevBras Est Pop.2009;26(1): 7-19.https://doi.org/10.1590/S0102-30982009000100002

45. Dias PC, Henriques P, Anjos LA, Burlandy L. Obesidade e políticas públicas: concepções e estratégias adotadas pelo governo brasileiro.Cad Saúde Pública. 2017;33(7):e00006016.http://dx.doi.org/10.1590/0102-311×00006016.

46. Ministério da Saúde. Caderneta da Gestante. $4^{a}$ ed. Brasília: Distrito Federal, 2018. [Acesso em 26 nov 2019].Disponível em: https://portalarquivos2.saude.gov.br/images/pdf/2018/agosto/31/Caderneta-da-Gestante-2018.pdf.

47. Portaria n 1.459, de 24 de junho de 2011. Institui, no âmbito do Sistema Único de Saúde - SUS - a Rede Cegonha. [Acesso em 26nov 2019]. Disponível em: https://www.as.saude.ms.gov.br/wp-content/uploads/2016/07/Portaria-GM-MS-n¹.459-dodia-24-de-junho-de-2011.pdf.

\section{Colaboradores}

Pires CC e Capelli JCS participaram da concepção do projeto, da análise, da interpretação dos dados, da redação e da revisão crítica do artigo. Carmo CN participou da análise, da interpretação dos dados e da revisão crítica do artigo. Carvalho MF, Lima FF, Monteiro LS, Sperandio N e Pereira S participaram da interpretação dos dados, redação do artigo e revisão crítica do artigo. Todos os autores aprovaram a versão final a ser publicada e são responsáveis por todos os aspectos do trabalho na garantia da exatidão e integridade de qualquer parte da obra.

Conflito de Interesses: Os autores declaram não haver conflito de interesses.

Recebido: 28 de janeiro de 2020

Aceito: 18 de abril de 2020 